\title{
Predisposing factors of rhinitis medicamentosa: what can influence drug discontinuation?*
}

\author{
Eugenio De Corso, Rodolfo Francesco Mastrapasqua, Laura Tricarico, Stefano Rhinology 58: 3, 233 - 240, 2020 \\ Settimi, Tiziana Di Cesare, Dario Antonio Mele, Lucrezia Trozzi, Giampiero ～https:/doi.org/10.4193/Rhin19.295 \\ Salonna, Gaetano Paludetti, Jacopo Galli \\ *Received for publication: \\ August 28, 2019 \\ "A. Gemelli" Hospital Foundation, IRCCS, Head and Neck Surgery Area, Institute of Otorhinolaryngology, Catholic University of the \\ Sacred Heart, Rome, Italy \\ Accepted: November 28, 2019
}

\begin{abstract}
Background: the primary end point of our study was to define risk factors and identify the underlying conditions that may have led to the abuse of vasoconstrictors in rhinitis medicamentosa. Moreover, we analysed factors that may influence the vasoconstrictors discontinuation.

Methodology: this was a prospective case-control observational study. Cases and controls were evaluated at the baseline in order define factors that may have influenced onset of rhinitis medicamentosa. They were re-evaluated at 3 months to verify symptoms control and drug discontinuation. Finally, they underwent a phone call questionnaire after 12 months regarding drug discontinuation. A potential bias of our study is that evaluating discontinuation we included subjects treated differently according to the main diagnosis.
\end{abstract}

Results: patients with rhinitis medicamentosa were more frequently smokers than controls, they had higher mean HAMA scores and positive psychiatric diseases history. Additionally, we frequently detected a local inflammation at nasal cytology in patients with rhinitis medicamentosa. A significant improvement in all nasal symptoms scores was observed in cases and controls but $29.4 \%$ of cases did not discontinue the vasoconstrictors. Two major factors negatively influenced discontinuation: positive nasal cytology and pathological HAMA score.

Conclusion: we observed that positive local inflammation, anxiety and smoking habit correlate positively with vasoconstrictors abuse. In addition, we demonstrated that anxiety and local inflammation were the most important factors impairing drug discontinuation.

Key words: anxiety, nasal cytology, nasal decongestants, rhinitis, smoking

\section{Introduction}

Nasal congestion may severely affect quality of life impairing social life, sleep, work, school and daily-life in general ${ }^{(1)}$; its prevalence is estimated to be up to $30 \%$ in the general population $^{(2)}$. Systemic and topical nasal decongestants are very often prescribed for the symptomatic treatment of acute sino-nasal conditions such as "common cold"; their efficacy has been clearly demonstrated but prompt resolution of nasal blocking and over-the-counter availability can frequently lead to inappropriate prescription renewals and excessive self-medication ${ }^{(3)}$. Rhinitis medicamentosa (RM), also called "rebound" or "chemical" rhinitis, is defined as a chronic dysfunction of the nasal mucosa secondary to prolonged use of topical vasoconstrictive agents (4). The term RM may be also used to describe the adverse nasal congestion that develops after using other medications including ACE-inhibitors, reserpine, guanethidine, phentolamine, methyldopa, prazosin, beta-blockers, chlorpromazine, aspirin, other NSAIDs and oral contraceptives ${ }^{(5)}$. There are differences between the mechanism through which nasal congestion can be caused by topical nasal decongestants and oral medications; hence we focus our attention only on the local ones.

Currently nasal vasoconstrictors are of two main classes: sympa- 
thomimetic amines and imidazole derivatives such as naphazoline, xylometazoline and oxymetazoline. Their prolonged use may lead to ischemia of the nasal mucosa, due to intense vasoconstriction, and consequently development of interstitial oedema ${ }^{(6)}$. Prolonged a2-receptors stimulation induces decreasing of receptors by downregulation. These effects would induce relative dilatation of submucosal sinusoid venous plexuses due to loss of dynamic venous vasoconstriction. Adrenergic receptors could also become refractory to nasal decongestants, causing the patient to increase the doses ${ }^{(7)}$.

Symptoms of RM include nasal congestion without rhinorrhoea, postnasal drip, sneezing following several days of decongestant use. If the vasoconstriction is prolonged, a compensatory reversal reaction i.e. secondary vasodilatation occurs, and the mucosa becomes increasingly less sensitive to subsequent applications of vasoconstrictors, resulting in drug habituation. Paradoxically, patients often try increasing both dose and frequency of nasal sprays which aids the onset of rhinitis medicamentosa and worsens the condition ${ }^{(8)}$. Patients with RM may develop psychological dependence and an abstinence syndrome consisting of headache, restlessness and anxiety after nasal decongestants discontinuation ${ }^{(9,10)}$.

The primary endpoint of our study was to define the predisposing factors of RM and identify the underlying conditions that may have led to the abuse of vasoconstrictors. Moreover, we analysed the clinical outcomes and factors that may influence the discontinuation of vasoconstrictors.

\section{Material and methods}

\section{Study design and study population}

This is a case-control observational study (evidence: level 3 ). We enrolled 68 cases (18-65 years; mean age $43 \pm 14 y$ ) complaining with persistent rhinitis and history of vasoconstrictors abuse for at least one month, consecutively diagnosed and followed-up, between June 2018-June 2019, at the Rhinology Centre of our Institution. As control group, we enrolled 51 patients affected by persistent rhinitis who denied the use of vasoconstrictors, (18-65 years, mean age $45 \pm 13 y$ ). The study was approved by the Ethics Committee of our Institution (ID2399). All patients were willing to participate and consented to their inclusion with formal written consent submitted to everyone when recruited.

The demographic characteristics of study population are summarized in Table 1.

\section{Inclusion and exclusion criteria}

Inclusion criteria were:

- Age: 18-65 years.

- Persistent symptoms of rhinitis dominated by nasal congestion in the last month.

- Continuous non-prescription use of topical vasoconstrictors for 1 or more applications per day for more than 1 months.

- Nasal congestion as major symptom, with median NOSEscore of at least 3 (scale $0-4$ ).

Exclusion criteria were:

- Acute and chronic rhinosinusitis with or without Nasal polyps.

- Local or systemic steroid treatment less than 4 weeks before the inclusion in the study.

- Previous septoplasty, rhinoplasty, endoscopic sinus surgery.

- Inferior turbinate decongestion during last year.

- Septal deviation requiring surgery.

- Current malignancy of any kind or previous radiotherapy for head-neck cancer.

- Pregnancy.

\section{Clinical investigation design}

Cases and controls were evaluated at the baseline (T0) and after 3 months (T1). At the baseline (T0) patients underwent investigations to phenotype their rhinitis as well as to define their underlying condition and risk factors for vasoconstrictor abuse. Patients were asked about their personal history and vasoconstrictors if used (type, duration and frequency of use etc). CT scan was also taken into consideration if performed previously. At T1, patients were clinically re-evaluated regarding the controls of symptoms, while only the cases were specifically investigated about vasoconstrictors discontinuation. Finally, the cases underwent a phone-call questionnaire 12 months (T2) after recruitment to review vasoconstrictor discontinuation anew. Baseline evaluation (TO):

- NOSE-scale made up of five symptom scores: congestion or stuffiness, blockage or obstruction, breathing through nose, trouble sleeping, trouble to get air through nose during exercise or exertion. Each symptom score is rated on a five-point categorical scale: not a problem (0), very mild problem (1), moderate problem (2), fairly bad problem (3), severe problem (4). The NOSE score was calculated as the mean of the five items investigated, and higher scores represent poorer outcome ${ }^{(11)}$.

- Hamilton anxiety rating scale (HAM-A) ${ }^{(12)}$ probes 14 parameters and takes 15-20 minutes to complete the interview and score the results. Each item is scored on a 5-point scale, ranging from $0=$ not present to $4=$ severe. The scale has proven useful not only in following individual patients but also in research involving many patients. HAMA scores correlate with severity of Anxiety (14-17=Mild; 18-24=Moderate; 25-30=Severe).

- Visual Analogical Scale (VAS), ranging 0-10 cm, scores the main nasal symptoms (nasal obstruction, rhinorrhoea, sneezing, nasal itching), ocular symptoms (redness/puffiness, watery eyes), other patient-reported outcomes (PROs) 
Table 1. Demographic data and disease profiling. all data are expressed as mean +SD. P-values are expressed if significant.

\begin{tabular}{|c|c|c|c|c|}
\hline & $\begin{array}{l}\text { All patients } \\
\quad N=119\end{array}$ & $\begin{array}{l}\text { Cases } \\
\mathrm{N}=68\end{array}$ & $\begin{array}{c}\text { Controls } \\
\mathrm{N}=51\end{array}$ & P value \\
\hline Age, years (mean $\pm S D$ ) & $43 \pm 14$ & $41 \pm 13$ & $46 \pm 14$ & n.s \\
\hline Gender (Females) & $47(39.5 \%)$ & $27(39.9 \%)$ & $20(39.2 \%)$ & n.s \\
\hline Smoking & $26(21.2 \%)$ & $43(65.2 \%)$ & $23(33.8 \%)$ & $<0.01$ \\
\hline Allergy & $38(31.9 \%)$ & $23(33.8 \%)$ & $15(29.4 \%)$ & n.s \\
\hline Positive cytology at baseline & $53(44.5 \%)$ & $37(54.4 \%)$ & $16(31.4 \%)$ & $<0.05$ \\
\hline Total score HAMA (mean \pm SD) & $13.4 \pm 10.2$ & $19.6 \pm 8.9$ & $3.87 \pm 0.5$ & $<0.01$ \\
\hline Duration of rhinitis, years (mean \pm SD) & $10.3 \pm 6.2$ & $10.0 \pm 4.3$ & $10.6 \pm 7.4$ & n.s \\
\hline Total Vas score (CSS) (mean \pm SD) & $41.5 \pm 20.2$ & $44.2 \pm 18.7$ & $37.9 \pm 22.2$ & n.s \\
\hline T4NSS (mean \pm SD) & $18.8 \pm 9.5$ & $18.2 \pm 9.0$ & $19.9 \pm 11.8$ & n.s \\
\hline T2OSS (mean \pm SD) & $5.4 \pm 7.5$ & $5.4 \pm 9.1$ & $5.4 \pm 4.7$ & n.s \\
\hline PROs (mean \pm SD) & $16.9 \pm 10.8$ & $20.1 \pm 10.8$ & $12.6 \pm 9.1$ & $<0.05$ \\
\hline Previous Turbinate Surgery & $28(23,5 \%)$ & $18(26.5 \%)$ & $10(19.6 \%)$ & n.s \\
\hline Psychiatric diseases history & $10(8.4 \%)$ & $9(13.2 \%)$ & $1(2 \%)$ & $<0.05$ \\
\hline
\end{tabular}

All data are expressed as mean \pm SD. P-values are expressed if significant. SD: standard deviation; HAMA: Hamilton anxiety rating scale; CSS: Composite symptom score; T4NSS: Total four main nasal symptom score; T2OSS: Total two ocular symptom score; PROs: Patients related outcomes.

such as difficulty going to sleep, night-time awakenings, nasal congestion on awakening ${ }^{(13)}$. Patients had to answer to the question: "How troublesome is your symptom?" (0:no symptoms; 10:symptom extremely bothersome). The following score were obtained:

- Total Nasal Symptom Score (T4NSS) adding scores of four main nasal symptoms,

- Total Ocular Symptom Score (T2OSS) adding scores of two main ocular symptoms,

- Total Symptom Score for PROs (TSS-PROs) adding the PROs,

- Composite Symptoms Score (CSS) adding the T4NSS, T2OSS, TSS-PROs scores.

- ENT evaluation with endoscopy to exclude septal deviation, nasal polyps and purulent rhinorrea.

- Nasal cytology was performed on nasal scraped tissue by rhinoprobe (Farmark s.n.c, Milan, Italy) on inferior turbinate bilaterally. The samples were spread on glass slides and immediately fixed in 95\% ethylalcohol and stained with MayGrunwald-Giemsa. Percentage of leukocyte was assessed by microscopic cytological examination as the mean of at least 10 reach fields observed. The slides were examined under oil immersion by light microscopy at a magnification of $x 1000$. Nasal cytology was supposed to be positive if an infiltrate of leukocyte was observed including eosinophils, neutrophils and mast cells.

- Allergic evaluation including allergic skin tests for common inhaled allergens (at least 18 including domestic powders, pollen, fungi, dogs, cats), determination of specific serum
IgE levels (RAST) and nasal provocation test. The last one was performed only in the case of negative Skin prick test and negative RAST to exclude local allergic rhinitis.

- In accordance with the ARIA project ${ }^{(14)}$, we divided patients into allergic/non-allergic. Non-allergic ones were divided on the basis of the presence/absence of inflammation during nasal cytology as a reflection from our previous experience $^{(15-17)}$. At baseline, a considerable amount of time was spent to explain the importance of drug discontinuation. We informed the patients about the causes of their rhinitis and that the overuse of vasoconstrictor affected negatively the resolution of the rhinitis; we emphasized that limiting its frequency and duration was central to avoid perpetuating of RM. They were given accurate information on the clinical findings from allergic evaluation and nasal cytology and were prescribed appropriate medical therapy. Allergic-rhinitis patients were prescribed hypertonic/isotonic saline irrigations and topical corticosteroids with local or systemic antihistamine, one or twice-per-day depending on the symptoms load. Non-allergic patients were treated according to their nasal cytological results; patients with local inflammation were treated with hypertonic/isotonic saline irrigations and topical corticosteroids, negative ones with hypertonic/isotonic saline irrigations and capsaicin.

- At the first follow-up (T1), patients underwent VAS-questionnaire, NOSE-scale as well as a specific consultation regarding the adherence to the prescribed therapy and discontinuation of vasoconstrictors. All cases, especially relapsing patients were recommended to avoid further use 


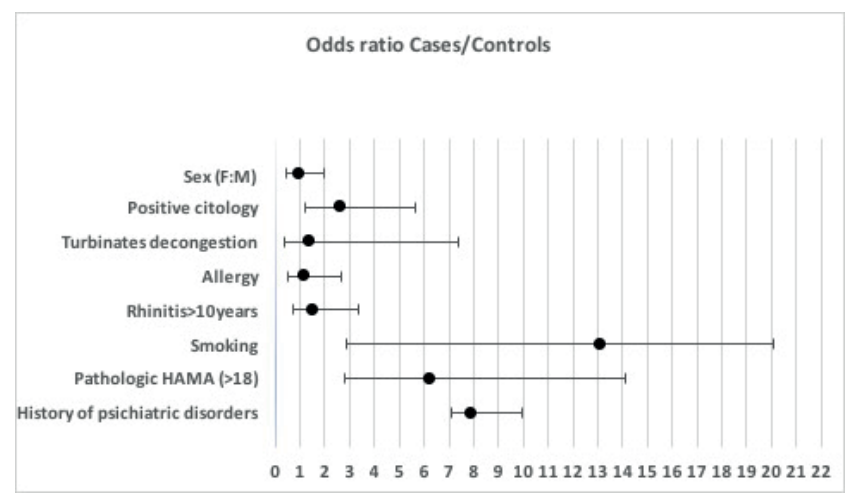

Figure 1. Forest Plot of Odds ratio of clinical factors involved in developing of RM, data are showed as ODDs ratio and confidence intervals.

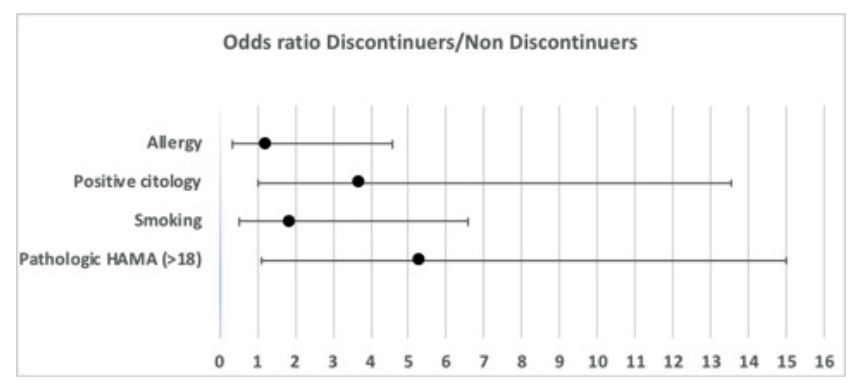

Figure 2. Forest Plot of Odds ratio of non-discontinuing vasoconstrictors, data are showed as ratio (dot) and confidence intervals.

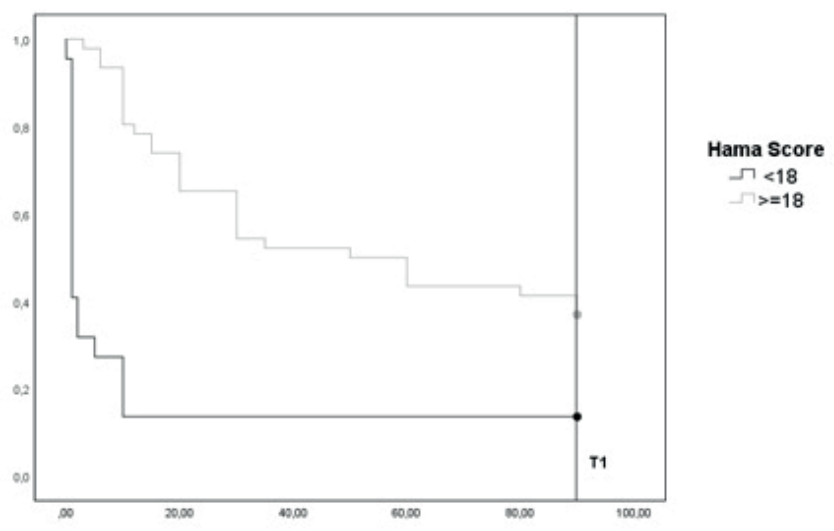

Figure 3. Cumulative probability for drug discontinuation in RM cases by Kaplan-Meier Method. In black are plotted patients with an HAMA score less than 18 , on light grey patients with a score higher than 18 . The difference between the two groups was significant at the Log Rank Test $(p<0.01)$.

of vasoconstrictors and adhere to nasal saline irrigations plus nasal corticosteroids or capsaicin if needed.

- At the conclusive phone-call follow-up (T2), patients were investigated about their therapy being taken over the follow-up time and the possible recurrence of vasoconstrictor abuse. Specific topics were: drug discontinuation, time until discontinuation, addiction relapse, time until addiction relapse. Patients were candidate to Psychologist evaluation if not discontinuing or relapsing. Patients were considered relapsing if recurring in daily use of vasoconstrictors.

\section{Statistical analysis}

Statistical analysis was performed by Microsoft-EXCEL and IBMSPSS-statistics ${ }^{(25)}$ software-package (Armonk, NY-IBMcorp). Data obtained by questionnaires were expressed as mean \pm Standard Deviation (SD). Mean questionnaires scores were compared by Mann-Whitney test for independent samples, $\chi^{2}$ was performed for probability of drug abuse recurrence and Fisher-test for risk of drug non-discontinuation. Kaplan-Meier function was applied for cumulative probability of discontinuation of vasoconstrictors. The limit for statistical significance was set at $\alpha=0.05$. Uninominal logistic regression analyses was performed for clinical features (allergy, positive nasal cytology, smoking, severity of symptoms, HAMA) associated to RM to establish the relative risk of developing RM. We considered that HAMA-questionnaire was significantly positive for values $>18$ including moderate and severe Anxiety. The regression analyses was performed also to establish the factors that may influence the discontinuation of the drug, considering both patients that did not discontinued vasoconstrictors at $\mathrm{T} 1$ and the ones that relapsed at $\mathrm{T} 2$.

\section{Results}

Clinical aspects of rhinitis medicamentosa

All cases completed the 3 months'follow-up and responded to the phone-call questionnaire. The most frequently abused vasoconstrictors were nafazoline [42 cases (65\%)] and oximetazoline [17 cases (26\%)]; there were very low percentages of other drugs as xilometazoline [ 3 cases (5\%)], efedrine [ 2 cases (3\%)] and tramazoline [1 case (1\%)]. The daily overuse of nasal decongestants ranged between 1-15 applications per day and the mean number of applications was 2.7 times. Investigating duration of vasoconstrictor abuse we found a large range: 1-240 months(mean: 31.83 months). Local inflammation was detected in $37 / 68$ of RM patients (54.4\%) and $16 / 51$ of controls (31.4\%) and the difference was statistically significant $(p<0.05)$. In the RM patients, 35/37(94\%) of them have shown a neutrophilic inflammation whereas in 2/37 patients (6\%), we observed an eosinophilic one. In the control population, 10/16 (62\%) have neutrophilic inflammation whereas $6 / 16$ (38\%) have an eosinophilic one. The differences were statistically significant $(p<0,05)$. Based on the baseline evaluation, we detected a coexisting allergic rhinitis in 23/68 (33.8\%), the remaining patients were negative to allergy test. Non-allergic patients have a negative nasal cytology in 21/45 (46,7\%) and positive in 24/45 (53.3\%). In control group, they were respectively $15 / 51$ (29.4\%), 24/51 (47.1\%) and 12/51 (23.5\%).

Clinical features were evaluated in the cases and controls and statistical comparisons were performed (Table 1). Clinically, both groups were homogenous with regards to symptom severity at baseline for T4NSS, T2OSS and CSS-score; a significant difference has been observed only for mean PROs (20.1 vs 12). Gender distribution between RM patients and controls showed 
Table 2. Baseline and 3-months follow-up Questionnaires score results.

\begin{tabular}{|c|c|c|c|c|c|c|}
\hline & \multicolumn{2}{|c|}{ Cases $\mathrm{N}=68$} & \multirow[t]{2}{*}{ P value } & \multicolumn{2}{|c|}{ Controls $\mathrm{N}=\mathbf{5 1}$} & \multirow[t]{2}{*}{ P value } \\
\hline & TO & T1 & & TO & T1 & \\
\hline Total Vas score (CSS) (mean \pm SD) & $44.0 \pm 18.27$ & $34.6 \pm 11.9$ & $p<0.01$ & $37.98 \pm 22.2$ & $31.11 \pm 11.63$ & $p<0.01$ \\
\hline T4NSS (mean \pm SD) & $18.8 \pm 9.0$ & $16.9 \pm 8.4$ & $\mathrm{p}<0.01$ & $19.9 \pm 11.8$ & $18.20 \pm 9.12$ & $\mathrm{p}<0.01$ \\
\hline T2OSS (mean \pm SD) & $5.3 \pm 9.1$ & $3.8 \pm 3.8$ & $\mathrm{p}<0.05$ & $5.4 \pm 4.7$ & $2.61 \pm 4.70$ & $p<0.01$ \\
\hline $\mathrm{PROs}($ mean $\pm \mathrm{SD})$ & $20.8 \pm 10.88$ & $12.82 \pm 8.1$ & $p<0.05$ & $12.6 \pm 9.1$ & $10.09 \pm 4.12$ & $p<0.05$ \\
\hline
\end{tabular}

Table 3. Stratification of patients basing on patologic $(>18)$ and normal $(<18)$ HAMA scores, all data are expressed as mean \pm SD. P values are expressed if significant.

\begin{tabular}{|c|c|c|c|c|}
\hline Rhinitis Medicamentosa patients & $\begin{array}{c}\text { All cases } \\
\mathrm{N}=68\end{array}$ & $\begin{array}{c}\text { Pathologic HAMA } \\
(>18) \\
N=46\end{array}$ & $\begin{array}{c}\text { Normal HAMA } \\
(<18) \\
\mathbf{N}=\mathbf{2 2}\end{array}$ & $P$ value \\
\hline Number of administration per day (mean days \pm SD) & $2.7 \pm 2.1$ & $2.8 \pm 1.4$ & $2.5 \pm 3.1$ & $<0.05$ \\
\hline Duration of vasoconstrictors abuse (mean months \pm SD) & $31.8 \pm 36.1$ & $36.9 \pm 41.3$ & $21.3 \pm 17.7$ & n.s \\
\hline Discontinuation ( $\mathrm{N}$ of patients) ${ }^{\circ}$ & $48(70.6 \%)$ & $29(63.0 \%)$ & 19 (86.4\%) & $<0.05$ \\
\hline Discontinuation time of vasoconstrictors (mean days $\pm \mathrm{SD}$ ) & $17.7 \pm 23.7$ & $28.1 \pm 25.8$ & $2.4 \pm 3.4$ & $<0.01$ \\
\hline T4NSS (mean \pm SD) & $18.8 \pm 9.1$ & $18.8 \pm 9.2$ & $18.7 \pm 9.1$ & n.s \\
\hline T2OSS & $5.4 \pm 9.1$ & $5.4 \pm 4.6$ & $5.1 \pm 14.7$ & n.s \\
\hline TSS-PROS & $20.1 \pm 10.1$ & $22.0 \pm 12.1$ & $16.3 \pm 6.2$ & n.s \\
\hline CSS & $44.2 \pm 18.3$ & $46.2 \pm 17.6$ & $40.1 \pm 19.3$ & n.s \\
\hline
\end{tabular}

*Number of days from visit at baseline and last administration of vasoconstrictor; ${ }^{\circ}$ Percentage of patients that interrupted vasoconstrictors after our intervention.

no significant differences, while RM patients were more likely to be smokers ( $35.3 \%$ vs $3.9 \%$; $P<0.01$ ). We also found significant differences between positive cytology $(54.4 \%$ vs $31.4 \%$; $\mathrm{P}<0.05)$, total HAMA mean score $(19.6 \pm 8.9$ vs $3.87 \pm 0.5 ; \mathrm{P}<0.01)$ and psychiatric diseases history ( $13.2 \%$ vs $2 \% ; \mathrm{P}<0.05)$. Allergic status was not different in cases and controls. We performed a univariate logistic regression analysis (Figure 1) describing factors that may be related to onset of RM: smoking (OR: 13.36; 95\% Cl: 2.98-59.8; $\mathrm{P}<0.01$ ), positive nasal cytology (OR: $2.61 ; 95 \%$ Cl: $1.21-5.58$; $\mathrm{P}<0.025)$, positive HAMA score (OR: 19.26 ; $95 \% \mathrm{Cl}$ : 6.71-55.16; $\mathrm{P}<0.01$ ) and history of psychiatric disorders (OR: 8.01; 95\% Cl: 7.11-9.98; $\mathrm{P}<0.01)$.

\section{Symptoms analyses and follow-up results}

We performed intra-group comparison of scores in cases and controls at baseline and at T1. A significant reduction was observed for all composite scores for both groups (Table 2 ). In the cases group, 48/68 (70.6\%) discontinued vasoconstrictors, while 20/68 (29.4\%) did not. In the group that continued with the use of vasoconstrictors, $5 / 20$ (25\%) did not discontinue the drug at $\mathrm{T} 1$ and were still using vasoconstrictors at $\mathrm{T} 2$; whereas 15/20 (75\%) discontinued the drug after T1 but relapsed at T2. Patients who did not discontinue the drug were recommended to undergo psychological evaluation; surgical option was also considered, specifically inferior turbinate decongestion.

\section{Stratification of cases by HAMA results and Discontinuation of vasoconstrictors}

We stratified cases based on the HAMA results: scores $>18$ were considered pathologic and $<18$ normal. We observed that RM patients with a pathologic HAMA-score had a higher mean of administrations per day ( $2.8 \pm 1.4$ vs $2.5 \pm 3.1 ; \mathrm{P}<0.05)$, a longer mean time needed for discontinuation ( $28.1 \pm 25.8$ vs $2.4 \pm$ 3.4 days; $\mathrm{P}<0.01)$ and were less likely to abandon the drug use (63.0\% vs $86.4 \% ; \mathrm{P}<0.01)$. However, we did not observe significant differences in the duration of vasoconstrictor abuse (36.9 \pm 41.3 vs $21.3 \pm 17.7$ months). Furthermore, we performed a symptom analysis in HAMApositive patients and negative ones. We did not observe a statistically significant difference in the mean T4NSS, T2OSS, TSS-PROs and CSS (Table 3). We did not find any significant difference between the type of vasoconstrictors used and the impact on discontinuation.

Analyzing factors that may influence discontinuation, we obtained Odds Ratio by Univariate Logistic Regression Analysis (Figure 2). Pathological HAMA score and positive findings at cytologic exam were significantly $(p<0.05)$ correlated to no drug 
Table 4. Logistic regression for drug discontinuation, all the predictors were entered as dichotomic variables except from Symptom Score. All variables were inserted in the same block. Significance threshold is 0.05 .

\begin{tabular}{|lccccc|}
\hline & B & S.E. & Wald & Sig. & OR \\
\hline Smoking habit & 0.612 & 0.648 & 0.890 & 0.345 & 1.844 \\
\hline Symptoms Score & 0.016 & 0.021 & 0.529 & 0.467 & 1.016 \\
\hline Allergy & 0.214 & 0.668 & 0.102 & 0.749 & 1.238 \\
\hline Hama $>18$ & 1.666 & 0.799 & 4.346 & 0.037 & 5.289 \\
\hline Positive Cytology & 1.306 & 0.662 & 3.895 & 0.048 & 3.692 \\
\hline
\end{tabular}

$B=$ coefficient of the regression for the variable; S.E. = Standard error; Wald $=$ Wald chi-square test results; Sig. $=$ statistical significance; $O R=$ Odd Ratio.

discontinuation, while smoking habit and positive prick-test show only a weak trend towards perpetration of drug abuse (not statistically significant). Symptoms severity score shows no trend towards either discontinuing the drug or not (Table 4).

We analysed the cumulative probability for drug discontinuation using the Kaplan-Meier method (Figure 3). In pathologic HAMA patients, the cumulative probability to discontinue the drug was lower than in normal HAMA patients. Long-rank test showed that the intergroup difference was significant $(p<0.01)$.

\section{Discussion}

RM is a condition that occurs most commonly in young and middle-aged adults with incidence reported to $1-9 \%$ of otolaryngology patients. However, these percentages may be underrepresentative of the true incidence due to the availability of over-the-counter medications ${ }^{(18)}$. Diagnostic criteria of RM were proposed in 1952: history of prolonged need for nasal medication, constant nasal obstruction, poor shrinkage of nasal mucosa on examination ${ }^{(19)}$. Since then, many criteria have been postulated to characterize RM based predominantly on retrospective studies. Nevertheless, validated criteria based on prospective studies are yet to be developed for a more sophisticated characterization of this condition ${ }^{(5)}$.

We performed an observational prospective study enrolling cases and controls homogeneous for age and gender. At baseline, both groups had comparable symptoms severity, specifically for T4NSS, T2OSS and CSS-score; a significant difference was observed only for PROs including difficulty going to sleep, night-time awakenings, nasal congestion on awakening etc. This suggests that sleeping disturbances related to persistent rhinitis is linked to a greater inclination to the abuse of vasoconstrictors with respect to the other symptoms.

Nasal congestion in subjects with presumed RM may be caused not only by the nasal decongestant use but also by co-morbid conditions such as allergic or non-allergic rhinitis and other nasal pathologies. Basing on our baseline evaluation, we documented coexisting allergic rhinitis in $33.8 \%$ of cases, whereas it was not possible establish the pre-existing proportion of nonallergic rhinitis respect the abuse of vasoconstrictors because not all the patients were followed at our institution before the baseline. Interestingly, we detected local inflammation more frequently in cases than in controls at the nasal cytology evaluation (54.4\% vs $31.4 \% ; \mathrm{P}<0.05)$. These results could be related not only to a persistent underlining rhinitis not responding to vasoconstrictors but also to the modifications induced by vasoconstrictors. Therefore, we strongly suggest a clinical re-evaluation in RM patients repeating allergy tests, endoscopy, nasal cytology etc in order to better define the underlining conditions that may have promoted the abuse of vasoconstrictors as well as to recommend tailored treatments.

Several studies have been performed to identify the onset of RM, nevertheless there is no agreement and more often the results are inconclusive. Some studies have demonstrated that rebound congestion does not develop with up to 8 weeks of topical decongestant use ${ }^{(20,21)}$, while others have suggested that the onset of RM occurs after 3 to 10 days of abuse recommending their discontinuation after 3 days ${ }^{(22)}$. This is supported by a study that shows increased nasal airway resistance after 3 days of daily or intermittent oxymetazoline ${ }^{(23)}$. On the other hand, Graf and Juto showed that rebound congestion does not begin until after 10 days of use in healthy volunteers and they observed that congestion continues to worsen from day 10 to day 30 24-26). Unfortunately, the harmful effects of prolonged treatment by vasoconstrictors on nasal mucosa i.e. from a mere rebound effect to a true RM, have only been described in a few studies in the literature(27). Knipping ${ }^{(28)}$, described the histological abnormalities on tissue samples of inferior turbinates from 22 patients with RM for at least 6 months revealing loss of ciliated cells, enlargement of intercellular spaces, thickening of the epithelial basement membrane, hyperplasia of goblet cells and a disturbed mucociliary clearance. Finally, a closer look the vascular endothelium revealed ultrastructural changes which explains the vascular permeability and the consequent interstitial edema. Suh et al. described earlier histological abnormalities in rabbits treated with phenylephrine, oxymetazoline or saline, observing sub-mucosal edema and loss of cilia on ulcerated respiratory epithelium after 2 weeks and more severe lesions after 4 weeks of use of nasal decongestants ${ }^{(29)}$. In this series, we included patients that were abusing vasoconstrictors for at least 1 months to ensure the inclusion of patients that may be considered true abusers and to exclude occasional ones. According to the literature ${ }^{(5)}$, the abuse of nasal decongestants may lead to damage of the epithelial barrier of the nasal mucosa, resulting in increased epithelial cell death. This leads to a heightened inflammatory cascade with exposure of tissue TLR receptors to pathogen-associated molecular patterns (PAMPs), 
thus attracting leukocytes into the nasal epithelium; this was observable under microscopy of the mucosal scrapings. With regards to the kind of inflammation in RM patients, we observed a predominant neutrophilic one; the percentage was significantly higher than the controls. The neutrophilic inflammation could be related to the increased permeability of epithelial barrier to pathogens, but further studies are required to confirm this. Even though it was not possible to establish the proportion of patients in which the local inflammation was pre-existing to vasoconstrictors abuse, these findings became particularly relevant analysing data in relation to the drug discontinuation. In fact, patients with local inflammation were less likely to discontinue the drug with respect to those without local inflammation. This findings encourage to detect the presence of local inflammation in abusing patients, as it seems to be related to a more aggressive phenotype in which patients are less prompt to discontinue the drug.

We paid particular attention to several psychological factors that could be involved in the natural history of RM. Adrenergic receptors usually become refractory to nasal decongestants, causing the patient to increase the doses (tachyphylaxis) ${ }^{(30)}$, looking for the rewarding effect of a temporary relief from nasal congestion; this could lead to addiction in some patients. In our analysis, the psychological factors showed significant differences between patients affected by RM versus controls. Smoking habit, history of psychiatric disorders, drug abuse, insomnia, anxiety disorders and depression, were present with a high prevalence among the patients affected by RM. We also found that patients with a pathological HAMA-score used nasal decongestant in higher doses/frequency per day than patients with a lower score and required a longer time to interrupt the abuse of vasoconstrictors. Our data is consistent with the suggestion that RM can be considered a true drug abuse, expressing the anxiety traits and impulse control issues as smoking ${ }^{(31)}$.

Finally, we obtain interesting results about discontinuation of the drug in the follow-up time after specific counselling and prescription of adequate medical therapy. We highlighted a strong correlation between anxiety trait and time needed to interrupt the abuse as well as the risk of relapse after interruption. The factors associated with difficult discontinuation were a HAMA-score above 18 (OR: 5,28; 95\% Cl:1.12-14.9; $\mathrm{P}<0.01$ ) and positive findings at cytological exam (OR: 3.6; $95 \% \mathrm{Cl}$ : 1.1-13.5; $\mathrm{P}<0.01)$. The smoking habit only showed a weak trend toward perpetration of drug abuse although this wasn't statistically significant. Moreover, we found that the symptoms severity showed no trend towards either discontinuing the drug or not which could be quite encouraging to the clinician for patient counselling, especially in cases of difficult discontinuation whereby the patient attributes the cause of abuse to symptoms severity. The knowledge of these factors from the findings mentioned above, could be a powerful tool for the clinician in the follow-up of patients affected by chronic rhinitis, especially to prevent drug addiction. A limitation of our study is related to a potential bias evaluating discontinuation. We included in fact in the analyses subjects treated differently according to the main diagnosis. It would have been preferable to have more homogeneous groups considering for example only subjects with no allergic rhinitis and reducing heterogeneity of the patients. Nevertheless, this is an observational study of patients followed in the daily clinical practice. We did not exclude allergic rhinitis because it may be one of the underlining factor very often observed in abusers of vasoconstrictors, furthermore we had opportunity to demonstrate that allergy was neither significantly related to the development of RM nor to the lower likelihood of drug discontinuation.

\section{Conclusion}

Resuming our most relevant results we detected more frequently local inflammation in RM patients than in controls, and it was related to a higher risk of not discontinuing the use of vasoconstrictors. RM patients were more frequently smokers even smoking had only a light impact on drug discontinuation. No specific rhinitis pattern especially the symptom severity was related to an increased risk of developing RM or its discontinuation; even sleeping disturbances seem induce patients to the abuse of vasoconstrictors more than other symptoms. An history of psychiatric disorders may be more likely to develop $\mathrm{RM}$ and in particular anxiety influences both the probability of developing an addiction to vasoconstrictors and discontinuation of vasoconstrictors.

\section{Acknowledgements}

The authors would like to thank Mr. Sanal Abraham (Catholic University of the Sacred Heart) for assistance with English revision of the text.

No funds were received for this research.

\section{Authorship contribution}

EDC: substantial contributions to conception and design, acquisition of data, and analysis and interpretation of data; main writer; gave final approval of this manuscript version to be published. RFM: acquisition of data, responsible of statistical analysis; drafting the manuscript; gave final approval of this manuscript version to be published. LT: data acquisition; database management; involved in drafting the manuscript; gave final approval of the version to be published. SS: data acquisition; database management; involved in drafting the manuscript; gave final approval of this manuscript version to be published; TDC: patients screening, baseline evaluation and informed consent; gave final approval of this manuscript version to be published; LT: patients screening, baseline evaluation and informed consent; gave final approval of this manuscript version to be 
published; DM: follow-up evaluations and phone calls; gave final approval of this manuscript version be published; GS: follow-up evaluations and phone calls; gave final approval of this manuscript version be published; GP: involved in drafting the manuscript or revising it critically for important intellectual content; gave final approval of this manuscript version to be published. JG: substantial contributions to conception and design; gave final approval of this manuscript version to be published.

\section{Conflict of interest}

The authors report no conflict of interest concerning the materials or methods used in this study or the findings specified in this paper.

\section{References}

1. Bousquet J, Duchateau J, Pignat JC, et al. Improvement of quality of life by treatment with cetirizine in patients with perennial allergic rhinitis as determined by a French version of the SF-36 questionnaire. J Allergy Clin Immunol 1996; 98(2): 309-16.

2. Åkerlund A, Millqvist E, Öberg D, Bende M. Prevalence of upper and lower airway symptoms: the Skövde population-based study. Acta Otolaryngol 2006; 26;126(5): 483-8

3. Eccles R, Martensson K, Chen SC. Effects of intranasal xylometazoline, alone or in combination with ipratropium, in patients with common cold. Curr Med Res Opin 2010; 12;26(4): 889-99.

4. Graf P. Rhinitis medicamentosa: aspects of pathophysiology and treatment. Allergy 1997; 52(40 Suppl): 28-34

5. Ramey JT, Bailen E, Lockey RF. Rhinitis medicamentosa. J Investig Allergol Clin Immunol 2006; 16(3): 148-55.

6. Graf P. Rhinitis medicamentosa: a review of causes and treatment. Treat Respir Med 2005; 4(1): 21-9.

7. Hein P, Michel MC. Signal transduction and regulation: Are all a1-adrenergic receptor subtypes created equal? Biochem Pharmacol 2007; 15; 73(8): 1097-106.

8. Black MJ, Remsen KA. Rhinitis medicamentosa. Can Med Assoc J 1980; 19; 122(8): $881-4$

9. Fleece L, Mizes JS, Jolly PA, Baldwin RL. Rhinitis medicamentosa. Conceptualization, incidence, and treatment. Ala J Med Sci 1984; $21(2):$ 205-8.

10. Snow SS, Logan TP, Hollender MH. Nasal spray "addiction" and psychosis: a case report. Br J Psychiatry 1980; 29; 136(3): 297-9.

11. Stewart MG, Witsell DL, Smith $T L$, Weaver EM, Yueh B, Hannley MT. Development and validation of the nasal obstruction symptom evaluation (NOSE) scale. Otolaryngol - Head Neck Surg. 2004; 130(2): 157-63.

12. Hamilton M. The assessment of anxiety states by rating. Br J Med Psychol 1959; 32(1): 50-5.

13. De Corso E, Anzivino R, Galli J, et al. Antileukotrienes improve naso-ocular symptoms and biomarkers in patients with NARES and asthma. Laryngoscope. 2019; 129(3): 551-7.

14. Brożek JL, Bousquet J, Agache I, et al.
Allergic Rhinitis and its Impact on Asthma (ARIA) guidelines-2016 revision. J Allergy Clin Immunol 2017; 140(4): 950-958.

15. De Corso E, Battista M, Pandolfini M, et al Role of inflammation in non-allergic rhinitis. Rhinology 2014; 52(2): 142-9.

16. De Corso E, Lucidi D, Battista $M$, et al. Prognostic value of nasal cytology and clinical factors in nasal polyps development in patients at risk: can the beginning predict the end? Int Forum Allergy Rhinol 2017; 7(9): 861-7

17. De Corso E, Bastanza G, Di Donfrancesco $V$, et al. Radiofrequency volumetric inferior turbinate reduction: long-term clinical results. Acta Otorhinolaryngol Ital 2016 36(3): 199-205.

18. Lockey R. Rhinitis medicamentosa and the stuffy nose. J Allergy Clin Immunol 2006; 118(5): 1017-8

19. Walker S. Rhinitis medicamentosa. J Allergy 1952; 23(2): 183-6.

20. Watanabe H, Foo TH, Djazaeri B, Duncombe P, Mackay IS, Durham SR. Oxymetazoline nasal spray three times daily for four weeks in normal subjects is not associated with rebound congestion or tachyphylaxis. Rhinology 2003; 41(3): 167-74.

21. Yoo JK, Seikaly H, Calhoun KH. Extended use of topical nasal decongestants. Laryngoscope 1997; 107(1): 40-3.

22. Åkerlund A, Bende M. Sustained Use of Xylometazoline Nose Drops Aggravates Vasomotor Rhinitis. Am J Rhinol 1991; 5(4): 157-60.

23. Morris S, Eccles R, Martez SJ, Riker DK, Witek TJ. An evaluation of nasal response following different treatment regimes of oxymetazoline with reference to rebound congestion. Am J Rhinol 1997: 11(2): 109-15.

24. Graf P, Juto JE. Decongestion effect and rebound swelling of the nasal mucosa during 4-week use of oxymetazoline. ORL J Otorhinolaryngol Relat Spec 1994; 56(3): 157-60.

25. Graf P, Juto JE. Correlation between objective nasal mucosal swelling and estimated stuffiness during long-term use of vasoconstrictors. ORL J Otorhinolaryngol Relat Spec 1994; 56(6): 334-9.

26. Graf P, Juto JE. Sustained use of xylometazoline nasal spray shortens the decongestive response and induces rebound swelling. Rhinology. 1995; 33(1): 14-7.

27. Mortuaire G, de Gabory L, François M, et al
Rebound congestion and rhinitis medicamentosa: Nasal decongestants in clinical practice. Critical review of the literature by a medical panel. Eur Ann Otorhinolaryngol Head Neck Dis 2013;1 30(3): 137-44.

28. Knipping S, Holzhausen HJ, Goetze G, Riederer A, Bloching MB. Rhinitis medicamentosa: Electron microscopic changes of human nasal mucosa. Otolaryngol Neck Surg 2007; 17; 136(1): 57-61.

29. Suh $\mathrm{SH}$, Chon KM, Min YG, Jeong $\mathrm{CH}$, Hong $\mathrm{SH}$. Effects of topical nasal decongestants on histology of nasal respiratory mucosa in rabbits. Acta Otolaryngol 1995; 115(5): 664-71.

30. Vaidyanathan S, Williamson P, Clearie K, Khan F, Lipworth B. Fluticasone Reverses Oxymetazoline-induced Tachyphylaxis of Response and Rebound Congestion. Am J Respir Crit Care Med 2010; 182(1): 19-24.

31. Conway KP, Swendsen JD, Rounsaville BJ, Merikangas KR. Personality, drug of choice, and comorbid psychopathology among substance abusers. Drug Alcohol Depend 2002; 65(3): 225-34

\section{Eugenio De Corso}

"A. Gemelli" Hospital Foundation IRCCS, Institute of Otorhinolaryngology

Head and Neck Surgery

Catholic University of the Sacred

Heart

Rome

Italy

Largo A. Gemelli n.1

00168 Rome

Italy

Tel: \pm 39630154439

Fax: \pm 3963051194

E-mail:

eugenio.decorso@policlinicogemelli.

it

eugenio.decorso@gmail.com 